\title{
DISEÑO Y APLICACIÓN DE UN MODELO DIDÁCTICO CON INNOVACIÓN EN MÉTODOS ACTIVOS PARA MEJORAR EL LOGRO DE CAPACIDADES EN LA ELABORACIÓN Y EJECUCIÓN DE PROYECTOS DE INVESTIGACIÓN CIENTÍFICA EN LOS ALUMNOS DE INGENIERÍA DE SISTEMAS UCV - TRUJILLO 2007
}

\section{DESIGN AND IMPLEMENTATION OF AN EDUCATIONAL MODEL WITH ACTIVE INNOVATION IN METHODS TO IMPROVE THA ACHIEVEMENT OF BUILDING IN THE PREPARATION AND IMPLEMENTATION OF RESEARCH PROJECTS IN SYSTEMS ENGINEERING STUDENTS UCV- TRUJILLO 2007}

\author{
Jorge Aranda G. \\ Universidad César Vallejo, \\ Trujillo, Perú
}

\section{RESUMEN}

La presente investigación surge de las deficiencias en la planificación y ejecución de proyectos de investigación que tienen los alumnos de la Escuela de Ingeniería de Sistemas. Este se refleja en la falta de coherencia en la redacción científica y en la asimilación de la metodología para elaborar la matriz de investigación, cuya consecuencia es que los estudiantes no desarrollan las capacidades investigativas que les permitirían elaborar proyectos.

Para enfrentar esta problemática se ha propuesto diseñar y aplicar un modelo didáctico basado en los métodos activos para la asignatura de metodología de la investigación que permita la elaboración y ejecución de proyectos de investigación científica.

El modelo didáctico denominado cíclico creciente, tiene un modelo pedagógico constructivista , cuyo objetivo es que los estudiantes alcancen niveles intelectuales superiores como el pensamiento crítico, la heurística, la innovación, la toma de decisiones y la solución de problemas. El modelo didáctico cíclico creciente pretende alcanzar competencias en los alumnos caracterizándose por una enseñanza constructora de sentidos desde el alumno, así como una enseñanza crítica y dialógica colectiva con una evaluación integral.

La ejecución del modelo didáctico fue posible a través de la guía de aprendizaje del curso de Metodología de la Investigación del área de Formación General de la Escuela de Ingeniería de Sistemas, la cual cuenta con 15 sesiones; se aplicó un pre test y un post test sobre las capacidades para elaborar y ejecutar proyectos de investigación, realizando la recolección de datos y el tratamiento respectivo, se llega a la conclusión que existe una influencia significativa en la mejora de dichas capacidades.

Palabras clave: modelo didáctico, capacidades, investigación, método activo.

\section{ABSTRACT}

The present investigation's work denominated arise of the deficiency in the planning and execution of investigation proyect that have the student of the school of system engineering, this is reflect for want of coherence in the redaction scientist and in the assimilation of the methodology for elaborate the investigation's matrix, because the students don't develop the investigation's capacitys that his permit elaborate projects.

For it is thought draw and apply a model didactic based in the active methods for the university course of investigation's methodology that permit the elaboration y execution of investigation's scientific projects. 
The model didatic denominated "Ciclic crescent" has a pedagogic model constructivist, his objetic is that the students reach level upper intellectuals, the same as critic thought, the heuristic, the innovation, the taking decision and the problem's solution.

The model didactic "ciclic crescent" seek to reach competitions in the students it is characterize for a teachiong builder of sense since the student, as soon as, a teaching criticism and dialogue collective with a integral evaluation.

The ejecution the model didactic "ciclic crescent" was possible though of the apprenticeship's guide of the university course of investigation's methodology of the area of formation general of the school of engineering's systems, it has 15 sessions, We applied a pre test and a post test about the aptitude for elaborate and execute investigation projects, realizing information's compilation and the respective treatment, I reach to conclusion that exist a significant influence in the improvement thes capacitys.

Keywords: Didactic model, capacities, investigation (research), active method.

\section{INTRODUCCIÓN}

El Perú se ubica en el puesto 114 (de 131) en los temas referidos a innovación y a la investigación en ciencia y tecnología, según reporte del World Economic Forum.

Según cifras del 2004, el Perú invierte 0,16\% del PBI en ID (Investigación y Desarrollo) y su gasto per cápita en este rubro es de US\$3,69 al año. Venezuela invierte US\$10,68 y Chile US\$39,45 [2], para el 2007 la inversión en I+D fue de $0,1 \%$ del PBI y para el 2009 es de $0,001 \%$ del PBI es decir 48 millones de un total de 72 mil millones, distribuidos en las diversas entidades como SENAMHI, CONCYTEC, IMARPE e INDEPA y otros según la revista electrónica Matices[1].

A nuestro país le hace falta invertir en diversas áreas, pero este trabajo está interesado en investigación y en educación. Es así que en educación solo invierte el $3 \%$ del PBI comparado con Chile y Argentina que es del $3,7 \%$ que en cantidad es USS 170 PPA mientras que Chile y Argentina invierten USS 402 y 465 PPA. [2].

La problemática se desarrolla en la Escuela de Ingeniería de Sistemas de la Universidad César Vallejo. En dicha escuela los trabajos de investigación que permitieron lograr la titulación de los egresados, en un porcentaje considerable contenían algunos errores en la elaboración de ciertos elementos de la investigación, sin embargo como en su mayoría eran investigaciones tecnológicas; las propuestas en su esencia no perdieron su veracidad o precisión al momento de abordar el problema. Dichas deficiencias demostraron la falta de habilidades para abordar la investigación.
El área de formación general está encargada de desarrollar la parte humanista de los estudiantes así como adaptarlos a la vida universitaria. Una de las asignaturas que desarrolla es la de Metodología de la investigación en el $\mathrm{V}$ ciclo. Sin embargo, la labor de esta asignatura no se ve complementada por las asignaturas de especialidad, porque se ha observado que la mayoría de ellas no plantean en forma lógica los elementos de investigación. [3]

Dentro de esta descripción académica encontramos las siguientes deficiencias de los alumnos de llevan el curso en el $\mathrm{V}$ ciclo. El primero es el bajo rendimiento en las evaluaciones escritas como se muestra en el siguiente grafico;

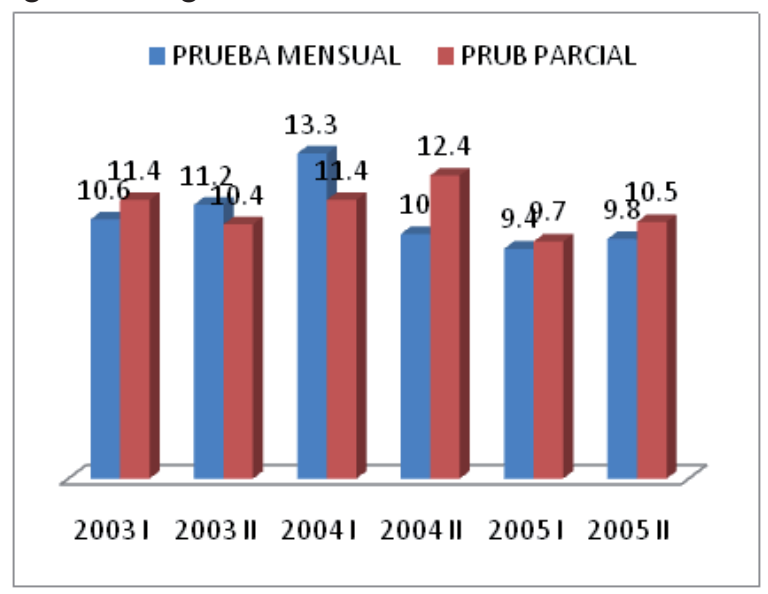

Figura 1. promedio de prueba mensual y promedio de prueba parcial en el curso de metodología de la investigación en la escuela de ing de sistemas según año de la UCV.

De continuar esta situación:

a) los índices de rendimiento en la asignatura no disminuirán es necesario mencionar que esto nada contribuye con las misiones de las escuelas que tienen como expectativa principal formar profesionales de calidad. 
b) cuando los alumnos lleguen al IX ciclo, que es cuando se elaboran proyectos de investigación para graduarse en su especialidad no tendrán las capacidades necesarias. De igual manera tendrán dificultades si piensan desarrollar alguna investigación.

c) los alumnos tendrán deficiencias para elaborar informes de investigación esto les traerá problemas cuando quieran participar en seminarios, congresos, o revistas científicas de su especialidad.

No existe un modelo didáctico que permita desarrollar las capacidades para elaborar y ejecuta con eficiencia los proyectos de investigación en estudiantes de Ingeniería de Sistemas de la universidad César Vallejo de Trujillo.

\section{Justificación}

El presente trabajo se justifica legalmente porque se está dando cumplimiento a las exigencias del curso de Metodología de la Investigación de la Escuela de Post grado de la UNPRG.

Desde el punto de vista académico se mejora el proceso enseñanza-aprendizaje ya que seria más eficiente en el logro de capacidades en investigación.

Por otro lado se justifica metodológicamente porque se quiere establecer pasos y criterios para la elaboración de un modelo didáctico para el aprendizaje.

Un modelo didáctico para formar investigadores es pertinente en estos tiempos donde las organizaciones tienen que aprender de sus experiencias es decir deben crear sus conocimientos. Si la universidad César Vallejo de Trujillo mejora eficientemente la formación de sus estudiantes entonces estará cumpliendo con su misión de formar profesionales para en el futuro.

Desde el punto de vista socialmente la universidad se beneficia por que se mejora la calidad en la formación de los estudiantes quienes van a desarrollar en un futuro próximo sus habilidades profesionales dentro de ellas las de investigador.

Nuestra motivación a investigar este tema responde o involucra aspectos como la interdisciplinariedad, los pilares de la educación y la sociedad del conocimiento.

\section{FORMULACIÓN DEL PROBLEMA}

Se observa deficiencias en la planificación y ejecución de proyectos de investigación, este se refleja en la falta de coherencia en la redacción científica, y en la asimilación de la metodología para elaborar la matriz de investigación por lo que los estudiantes no desarrollan las capacidades investigativas que les permiten elaborar proyectos.

\section{Definición del modelo didáctico "cíclico creciente".}

Un modelo es una herramienta conceptual para entender mejor un evento, es la representación del conjunto de relaciones que describen un fenómeno [4] .

Por modelo didáctico se entiende una representación selectiva de los elementos esenciales del fenómeno didáctico, que nos permite describirlo y explicarlo en profundidad, una ordenación racional que interpreta explica y dirige los fenómenos del aula desde un teoría subyacente (paradigma conductual, cognitivo, contextual). El modelo didáctico es intermedio entre el paradigma o teoría y la forma de hacer practica o método didáctico.

Los modelos didácticos son las representaciones valiosas y clarificadoras de los procesos de enseñanza-aprendizaje, que facilita su conocimiento y propician la mejora de la practica, al seleccionar los elementos mas pertinentes y descubrir la relación de interdependencia que se da entre ellos.

El modelo didáctico es una representación aproximada de la realidad se supone un alejamiento o distancia de la misma. Se caracteriza por ser aproximativa, provisional y no exclusiva. El modelo didáctico define los componentes de la acción de enseñar o educar y las acciones o tomas de posición que se realiza en torno a los componentes.

\section{Estructura de un modelo didáctico "cíclico creciente"}

La estructura de un modelo didáctico según Nemesio Núñez (2006) se puede presentar en el siguiente cuadro: 
Tabla 1: El modelo didáctico y sus principales elementos.

\begin{tabular}{|c|c|c|}
\hline \multirow{3}{*}{$\begin{array}{l}\text { 1.FUNDA- } \\
\text { MENTOS } \\
\text { TEORICOS } \\
\text { CIENTIFI- } \\
\text { COS }\end{array}$} & \multicolumn{2}{|c|}{ 1.1 Modelo pedagógico } \\
\hline & \multicolumn{2}{|c|}{ 1.2 Modelo Curricular } \\
\hline & \multicolumn{2}{|c|}{ 1.3 Concepción didáctica } \\
\hline \multirow{4}{*}{$\begin{array}{l}\text { 2.CARACTE- } \\
\text { RÍSTICAS }\end{array}$} & \multirow{3}{*}{ 2.1.Sujetos } & Alumnos \\
\hline & & Docente \\
\hline & & $\begin{array}{l}\text { Profesor- } \\
\text { Alumno }\end{array}$ \\
\hline & 2.2.Procesos & $\begin{array}{l}\text { Métodos, } \\
\text { capacidades, } \\
\text { procedimientos } \\
\text { de evaluación }\end{array}$ \\
\hline $\begin{array}{l}\text { 3.SECUEN- } \\
\text { CIA } \\
\text { DIDÁCTICA }\end{array}$ & \multicolumn{2}{|c|}{$\begin{array}{l}\text { Fases o etapas } \\
\text { Sesión de clase }\end{array}$} \\
\hline
\end{tabular}

Fuente: Núñez Nemecio (2006) "Ponencia: Elaboración de modelos didácticos II Congreso de investigación UNPRG sede Trujillo.

Según Dionicio (2007) [5] un modelo didáctico contiene elementos como: conceptualización, características fundamentos pedagógicos, procesos, elementos didácticos y secuencia

\section{Metodología}

Es un estudio cuantitativo con un diseño preexperimental de pre y post prueba. De acuerdo al propósito es un estudio de investigación aplicada.

Se utilizo una muestra única no aleatoria de 47 sujetos del V ciclo de Ing. De sistemas.

\section{Procedimiento}

1. Realizar un diagnostico de las habilidades de los estudiantes para elaborar y ejecutar proyectos de investigación científica cuantitativa y cualitativa. Usando la observación de evaluaciones y tesis.

2. Elaboración del modelo didáctico para elaborar yejecutarproyectos deinvestigación partir de información bibliográfica.

3. Aplicar el modelo didáctico a los estudiantes de ingeniería de sistemas, mediante sesiones consecutivas de aprendizaje.

4. Evaluar el impacto o efecto del modelo didáctico para mejorar el logro de las capacidades en la elaboración y ejecución de proyectos de investigación utilizando las técnicas estadísticas descriptivas, de frecuencias y pruebat.

5. Elaborar conclusiones y recomendaciones a partir de los resultados cuantitativos.

\section{RESULTADOS Y DISCUSIÓN}

Al revisar los exámenes escritos al final del año 2006 se observa los siguientes síntomas entre ellos:

1) Aún después de desarrollar el curso un $41 \%$ de alumnos no identifica y no relaciona las variables dependiente e independiente en enunciados explicativos.

2) El $20 \%$ de alumnos al terminar la asignatura no discrimina los paradigmas de investigaciones posibles que no tengan un aprendizaje significativo.

3) Más del $39 \%$ de alumnos desconoce la diferencia entre investigación descriptiva y explicativa.

4) Falta de coherencia entre los elementos del proyecto: los estudiantes no relacionan eficientemente objetivo-hipótesis- problema es decir no establecen una relación lógicas en la redacción. Aún permanece un $25 \%$ de alumnos que presenta estas incoherencias en sus formulaciones.

5) Deficiencia para elaborar ejemplos: mayormente se limitan a repetir los ejemplos de clase, no hay esa originalidad - capacidad creativa. Por lo tanto se debe trabajar con casos, de tal manera que poco a poco irán mejorando su nivel cultural. Es posible que la metodología que empleó el docente no sea la adecuada.

6) Uso de lenguaje común y no científico: los estudiantes en la elaboración de sus definiciones $o$ argumentos no se auxilian de un lenguaje científico, de acuerdo al nivel de la asignatura; mostrando pobreza lingüística.

7) Carencia de significado de términos: los alumnos aún no logran asimilar la terminología epistemológica tal como: experiencia, racionalidad, variables científica etc. Dado el nivel de complejidad del curso.

8) Confusiones en la redacción de los elementos de la investigación: no asimilan los criterios metodológicos para formular los objetivos, hipótesis, justificación entre otros. El $37 \%$ los aprenden en un momento pero no queda para su vida.

Las circunstancias anteriores explicadas nos permiten hacer un llamado a la creatividad de la pedagogía universitaria para disminuir significativamente estas carencias por ser un rol inherente al maestro universitario y por 
cultivar la investigación en sus estudiantes como herramienta de su futura vida profesional. De igual manera se realizó una observación a las tesis de ingeniería de sistemas que se encuentran en la biblioteca desde el 2001 hasta el 2005 llegando a las siguientes resultados según el gráfico 2 :

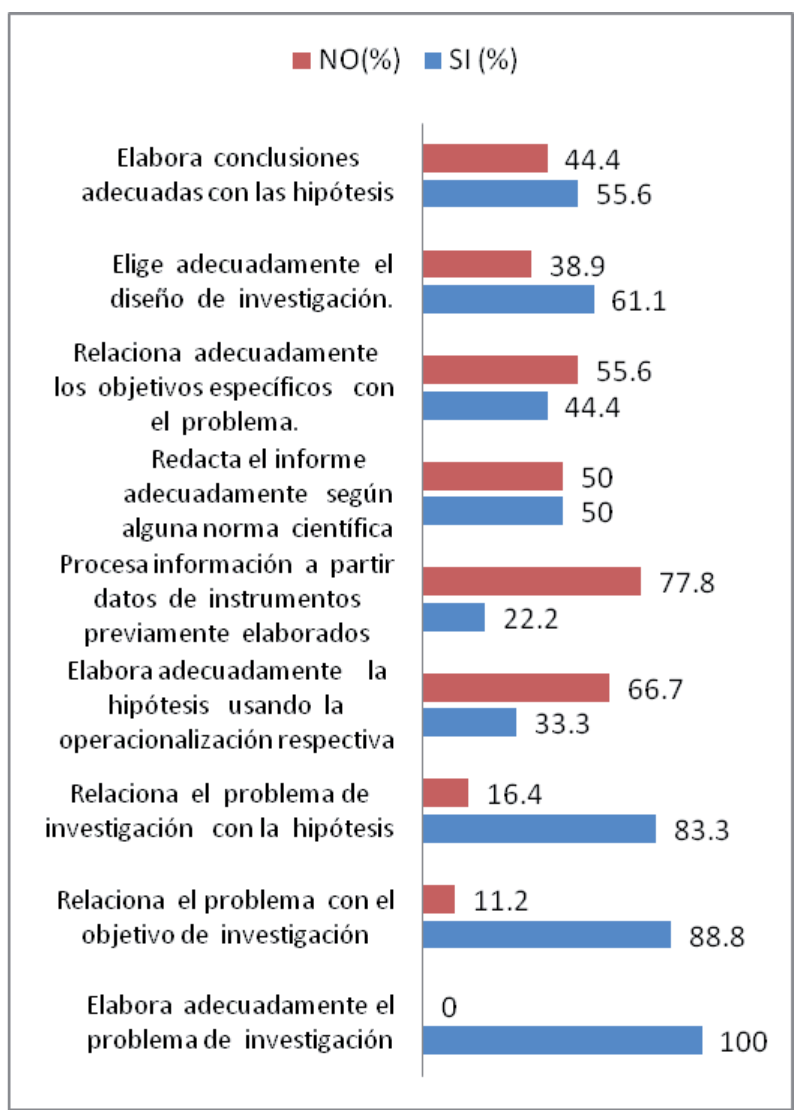

Figura 2. Gráfico de frecuencias relativas de los indicadores sobre los elementos de investigación en las tesis de los ex alumnos de ingeniera de sistemas UCV Trujillo $2002-2005$

\section{Denominación del modelo didáctico "cíclico creciente"}

Se tomo el nombre de cíclico creciente porque el modelo didáctico está estructurado en base a tres niveles de investigación , explorativo, descriptivo y explicativo: de tal manera se vuelve cíclico pero con mayor complejidad en cada nivel.

\section{Fundamentos teóricos científicos del} modelo didáctico "cíclico creciente"

\section{a) Modelo pedagógico}

El presente modelo didáctico responde a una pedagogía constructivista, que tiene por meta en los alumnos que accedan a niveles intelectuales superiores como el pensamiento critico, la heurística, la innovación, la toma de decisiones y la solución de problemas.

Donde la función del maestro es la de un facilitador, estimulador del desarrollo, con trato horizontal a sus discípulos, con énfasis en el estudio productivo, con una evaluación integral por procesos y sumativas. En general la unión de algunos rasgos de dos modelos uno progresista y el otro social.

\section{b) Modelo curricular}

El modelo curricular organizado por procesos cognitivos subjetivos es decir por competencias que se organizan en áreas de un currículo donde se busca formar estudiantes que problematicen su realidad y generen alternativas de cambio en su medio. Las competencias se organizan en capacidades y actitudes. Dichos profesionales practican valores positivos y humanistas.

\section{c) Concepción didáctica}

Para Contreras (1990) la didáctica se define como la disciplina que explica los procesos de enseñanza aprendizaje de acuerdo a la realización de los fines educativos. Supone entonces una mirada autorreflexiva vinculada con el compromiso moral (axiológica), así como una dimensión proyectiva (teleológica). [6].

La concepción didáctica de esta área se caracteriza por una enseñanza constructora de sentidos desde el alumno así como una enseñanza crítica y dialógica colectiva. Considerando una evaluación integral.

El modelo llamado "aproximativo o constructivo" (centrado en la construcción del saber por el alumno). Se propone partir de modelos, de concepciones existentes en el alumno y ponerlas a prueba para mejorarlas, modificarlas, o construir nuevas. [7]

El maestro propone y organiza una serie de situaciones con distintos obstáculos (variables didácticas dentro de estas situaciones), organiza las diferentes fases (acción formulación, validación, institucionalización), organiza la, comunicación de la clase, propone en el momento adecuado los elementos convencionales del saber (notaciones, terminología).

El alumno ensaya, busca, propone soluciones, las confronta con las de sus compañeros, las defiende o las discute. El saber es considerado en lógica propia. [8]. 


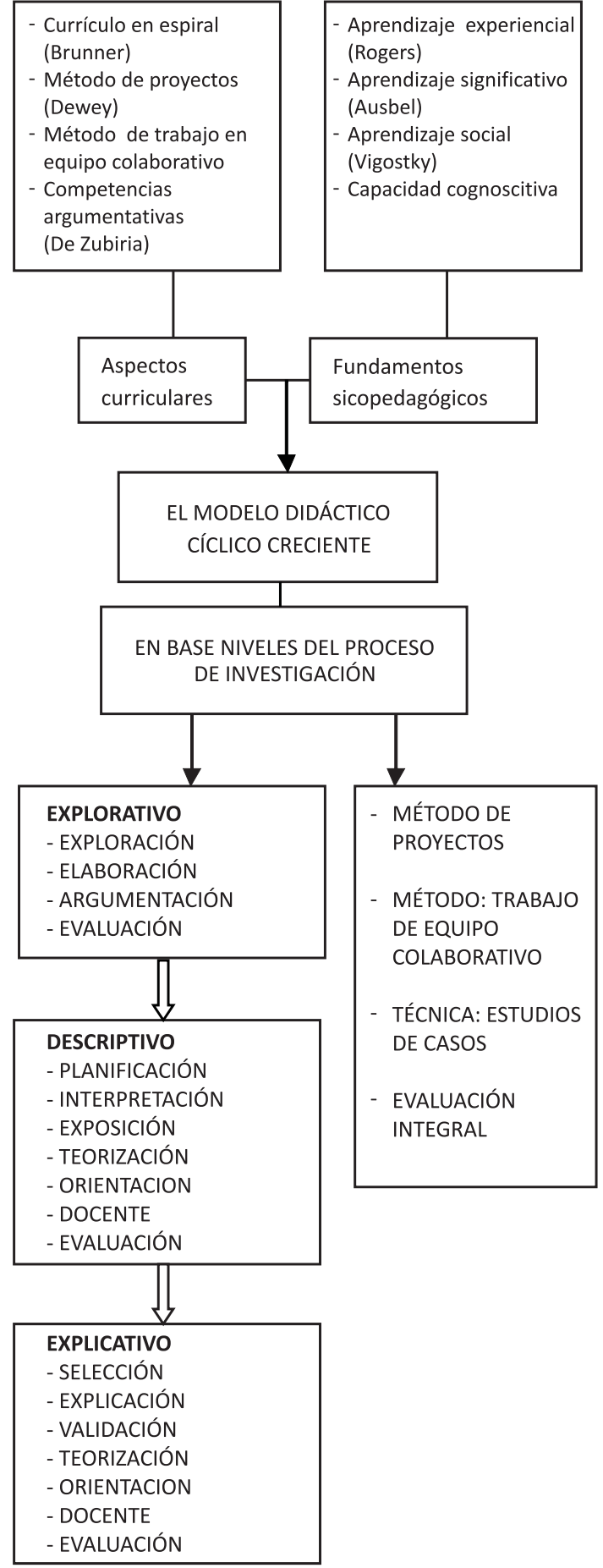

Figura 3. Representación del modelo didáctico cíclico creciente con sus fundamentos, niveles $y$ estrategias.

\section{RESULTADOS Y DISCUSION}

Luego de desarrollar el modelo didáctico en las unidades de análisis se obtienen los siguientes resultados luego de aplicar el pre test y el post test

\section{Resultado del pre test}

Luego de aplicar el pre test con preguntas abiertas o cerradas los resultados son los siguientes:
Tabla 1. Tabla de frecuencias absolutas y relativas de la aplicación del pre test sobre los niveles de las capacidades en la elaboración y ejecución de proyectos de investigación de los alumnos de ingeniería de sistemas 2007 II.

\begin{tabular}{|c|l|c|c|}
\hline $\begin{array}{c}\text { NIVELES } \\
\text { DE } \\
\text { LOGRO }\end{array}$ & $\begin{array}{c}\text { ESCALA } \\
\text { CUALITATIVA }\end{array}$ & $\begin{array}{c}\text { FRECUENCIAS } \\
\text { ABSOLUTAS }\end{array}$ & $\begin{array}{c}\text { FRECUENCIAS } \\
\text { RELATIVAS }\end{array}$ \\
\hline \multirow{2}{*}{ LOGRADO } & $\begin{array}{l}\text { MUY } \\
\text { BUENO }\end{array}$ & 0 & 0 \\
\cline { 2 - 4 } & BUENO & 2 & 4 \\
\cline { 2 - 4 } & REGULAR & 4 & 9 \\
\hline \multirow{2}{*}{$\begin{array}{c}\text { NOG } \\
\text { LOGADO }\end{array}$} & MALO & 33 & 70 \\
\cline { 2 - 4 } & MUY & 8 & 17 \\
\hline \multicolumn{2}{|c|}{ TOTAL } & 47 & 100 \\
\hline
\end{tabular}

Fuente: Propia del autor

Se puede observar que el $87 \%$ de alumnos tienen un puntaje Malo lo cual implica que la mayoría de alumnos carecen o no lograron hasta el momento las capacidades de elaboración y ejecución de proyectos de investigación, y que existen muy contados alumnos que poseen estas capacidades $(13 \%)$.

\section{Resultados del post test}

Tabla 2. Tabla de frecuencias absolutas y relativas de la aplicación del post test sobre los niveles de logro de las capacidades para elaborar y ejecutar proyectos de investigación de los alumnos de ingeniería de sistemas 2007 II.

\begin{tabular}{|c|l|c|c|}
\hline $\begin{array}{c}\text { NIVELES } \\
\text { DE } \\
\text { LOGRO }\end{array}$ & $\begin{array}{c}\text { ESCALA } \\
\text { CUALITATIVA }\end{array}$ & $\begin{array}{c}\text { FRECUENCIAS } \\
\text { ABSOLUTAS }\end{array}$ & $\begin{array}{c}\text { FRECUENCIAS } \\
\text { RELATIVAS }\end{array}$ \\
\hline \multirow{2}{*}{ LOGRADO } & $\begin{array}{l}\text { MUY } \\
\text { BUENO }\end{array}$ & 0 & 0 \\
\cline { 2 - 4 } & BUENO & 18 & 38 \\
\cline { 2 - 4 } & REGULAR & 26 & 55 \\
\hline \multirow{2}{*}{$\begin{array}{c}\text { NO } \\
\text { LOGRADO }\end{array}$} & MALO & 3 & 7 \\
\cline { 2 - 4 } & MUY & 0 & 0 \\
\hline \multicolumn{2}{|c|}{ TOTAL } & 47 & 100 \\
\hline
\end{tabular}

Fuente: Propio del autor 
se observa según la gráfica anterior que el $93 \%$ de los alumnos de ingeniería de sistemas se ubica entre bueno y regular lo cual se considera como capacidades logradas en la elaboración y ejecución de proyectos de investigación y que aun hay un $7 \%$ que no logro dichas capacidades.

\section{Análisis usando prueba $\mathbf{t}$}

Tabla 3. Comparación de valores de técnicas estadísticas entre el pre test y el post test.

\begin{tabular}{|l|l|}
\hline PRE TEST & POST TEST \\
\hline PROMEDIO = 8.1 & PROMEDIO=12.7 \\
DESVIACION & DESVIACION \\
ESTANDAR= 2.61 & ESTANDAR= 1.73 \\
$\mathrm{N}=47$ & $\mathrm{~N}=47$ \\
\hline
\end{tabular}

Hipótesis de investigación $\mathrm{Hi}$

$\mathrm{Hi}$ : Las puntuaciones en el logro de capacidades en el post test es mayor que en el pre test en los alumnos de ingeniería de sistemas.

\section{Hipótesis nula Ho}

Ho : Las puntuaciones en el logro de capacidades en el post test no es mayor que en el pre test en los alumnos de ingeniería de sistemas. $t=10.07$

Con g. L. $=47+47-2=92, y$ con 0.05 de error se tiene $t=1,66$ en la tabla de la prueba t. "t" experimental es mayor que "t" de tabla.

Luego se acepta la hipótesis de investigación Hi que dice: "Las puntuaciones en el nivel de logro de capacidades en el post test es mayor que en el pre test en los alumnos de ingeniería de sistemas".

Se conoce que toda aplicación de programas genera un impacto positivo en las unidades de análisis. En nuestro caso no es excepción, nuestro modelo didáctico según los niveles de escala cualitativa de nuestra variable dependiente tenemos que "el logro de capacidades en la elaboración y ejecución de proyectos de investigación científica alcanza un $93 \%$ entre bueno y regular en comparación con el pre test que solo llega al $13 \%$ es decir hay un incremento de $80 \%$ en el post test.

Además comparando la moda 6 y mediana 8 del pre test en comparación con los valores del post test moda 11 y mediana 13 observamos que existe un incremento en la moda es decir la mayoría de estudiantes tiene una nota regular de 11 incrementando 5 puntos. En cuanto a la mediana ahora tenemos que el $50 \%$ de estudiantes tenia una calificación de 08 que luego asciende a 13 , un incremento de 5 puntos.

Por otro lado el logro de capacidades se puede analizar se pueden también inferir según al escala cuantitativa (0 a 20) de la tabla $\mathrm{N} 08$ en donde el promedio del post test es 12.7 contra 08.1 del pre test.

Consideramos una mejora del logro de capacidades cuando un estudiante pasa de un nivel bajo o desaprobado a un nivel regular o bueno. La prueba $t$ nos demuestra que existe realmente una mejora significativa en el logro de capacidades de elaboración y ejecución de proyectos de investigación.

Cueva Valverde (1997) [9].realizo un estudio que buscaba el aprendizaje significativo a partir de un modelo instruccional, Zarate(1998) [10]. De igual manera mide la eficiencia del método funcional dinámico bajo un enfoque constructivista; en comparación con el presente trabajo que trata de ir mas allá hacia el aprendizaje por niveles de complejidad, utilizando una serie de fundamentos, como el currículo por espiral, el método colaborativo y otros.

El modelo didáctico cíclico creciente también evalúa competencias dentro de ellas capacidades y actitudes, es así que el trabajo en equipo, la actitud crítico reflexivo, demostrar honestidad y responsabilidad son las actitudes que cultiva, estas se encuentran evaluadas en los trabajos de equipo que desarrollan los alumnos obteniéndose resultados positivos de sus logros.

\section{CONCLUSIONES}

1. El modelo didáctico "Cíclico creciente" para la asignatura de Metodología de la Investigación mejora significativamente los niveles de logro de las capacidades en la elaboración y ejecución de proyectos de investigación de un $13 \%$ de logros iníciales a un $93 \%$ de logro del total de los alumnos del $V$ ciclo de la Escuela de Ingeniería de Sistemas de la UCV de Trujillo. 
2. El modelo didáctico "cíclico creciente" mejora el logro de capacidades en la elaboración y ejecución de 'proyectos de investigación en un $80 \%$ de alumnos del $V$ ciclo de ingeniería de sistemas.

3. El método "Trabajo de equipo- Colaborativo", y la guía de aprendizaje programada contribuyen con el modelo para lograr las capacidades en la elaboración y ejecución de proyectos de investigación de los alumnos del $\mathrm{V}$ ciclo de ingeniería de sistemas y es así como ellos también lo califican.

4. El modelo permitió acceder a niveles intelectuales superiores como el pensamiento critico, toma de decisiones, solución de problemas y la innovación, donde el profesor es un facilitador y estimulador del desarrollo con un trato horizontal a los alumnos.

5. Por modelo didáctico se entiende una representación selectiva de los elementos esenciales del fenómeno didáctico, que nos permite describirlo y explicarlo en profundidad, una ordenación racional que interpreta explica y dirige los fenómenos del aula desde un teoría y que comprende: fundamentos pedagógicos, características, métodos, rol e interacción alumno- maestro, y secuencia operativa grafica, en curso de pregrado a través del presente modelo didáctico denominado."Cíclico creciente" los resultados lo demuestran así.

\section{AGRADECIMIENTOS}

A la escuela de ingeniería de sistemas, al Mg Carlos Minchón, y en especial a la Dra. Emilia Urbina Gambini del área de formación general de la Universidad Cesar Vallejo de Trujillo Perú.

\section{REFERENCIAS}

[1] VEGA, Mariana, articulo "Apenas el 0,1\% del PBI anual se destina a la investigación" publicado en http://www.elcomercio.com pe/edicionimpresa/Html/2007-11- 6, 2007

[2] VÁSQUEZ, Rocy entrevista a José Luís Herce Vigil especialista en propiedad intelectual para América latina publicada en http://www.universia edu.pe/ eventos/universidadempresa.

2009.

[3] CURRÍCULO DE FORMACIÓN GENERAL,Universidad Cesar Vallejo Trujillo. Editorial vallejiana. Perú 2004.

[4] FLÓREZ, Rafael Evaluacion Pedagogica y Cognición .Editorial Mac Graw Hill Mexico.2003

[5] DIONICIO Víctor Modulo de seminario de tesis. Escuela de post grado Universidad Cesar Vallejo. Trujillo. Perú. 2007.

[6] CONTRERAS Domingo J, "La didáctica y los procesos de enseñanza aprendizaje", en „Enseñanza, Currículum y Profesorado. Introducción a la didáctica”, Akal, Madrid. 1990

[7] www.wikipedia.com biblioteca virtual consultado el 2/12/08.

[8] ORTíZ, Alexander Luís. La Activación del Proceso Pedagógico Profesional.

Pedagogía 97. La Habana. 1997

[9] Cueva Valverde, tesis "Modulo instruccional basado en enfoque constructivista y el logro de aprendizaje significativo en estudiantes de educación superior en la asignatura de metodología de la investigación". Escuela de post grado UNT Trujillo1997

[10] Zarate Iparraguirre, tesis "Método Funcional Dinámico para la enseñanzaaprendizaje de investigación educativa en el nivel superior no universitario" de la Escuela de Postgrado de la Universidad Nacional de Trujillo. 1998.

E-mail: jaranda@ucvvirtual.edu.pe 